\title{
Percutaneous Achilles tenotomy under local anaesthetic in the clubfoot clinic was safe during the COVID-19 pandemic, for both children and parents
}

\author{
Benjamin Barkham ${ }^{1} \cdot$ Thomas McNally $^{1} \cdot$ Aishling Russell ${ }^{2} \cdot$ Anna Bridgens $^{1} \cdot$ Yael Gelfer $^{1,3}$ [C]
}

Received: 20 May 2021 / Accepted: 14 June 2021 / Published online: 4 July 2021

(c) SICOT aisbl 2021

\begin{abstract}
Purpose An Achilles tenotomy is routinely required to correct the equinus deformity in Congenital talipes equinovarus (CTEV) patients as part of the gold standard treatment using the Ponseti method. This procedure can be performed in clinic under local anaesthetic or in theatre under general anaesthetic. The COVID-19 pandemic reduced theatre capacity and caused a delay to CTEV patients' treatment. A new standard operating procedure that allowed the tenotomies to be performed under local anaesthetic in the clinic was introduced. This study was looking into the safety, feasibility and parents' perspective of this procedure.

Methods The study was prospectively registered as a service improvement project and followed the SQUIRE guidelines (Ogrinc et al. in BMJ Qual Saf 25:986-992, 2016). All consecutive patients requiring a tenotomy were included. Data was collected prospectively including demographics, Pirani score and a carers' satisfaction questionnaire.

Results Twenty five patients (36 tenotomies) were included in the study. The median age was 9 weeks. All patients achieved ankle dorsiflexion of greater than $15^{\circ}$ post-op. None of the patients nor their parents contracted the COVID 19 virus. All parents reported a positive experience and 99\% felt less anxious about having the tenotomy done in clinic rather than theatre.

Conclusions The new service offering clinic tenotomies was found to be safe and clinically successful. This study is the first to show parents preference and excellent satisfaction with a tenotomy performed under local anaesthetic. The service has improved the allocation of resources and due to its success, will continue beyond the pandemic.
\end{abstract}

Keywords Clubfoot $\cdot$ COVID-19 $\cdot$ Tenotomy $\cdot$ Local anaethesia

Level of Evidence: IV

Yael Gelfer

yaelgelfer@gmail.com; yael.gelfer@stgeorges.nhs.uk

Benjamin Barkham

Benjamin.barkam@nhs.net

Thomas McNally

thomas.mcnaly2@nhs.net

Aishling Russell

Aisling.Russel@stgeorges.nhs.uk

Anna Bridgens

Ann.Bridgens@stgeorges.nhs.uk

1 Trauma and Orthopaedic Department, St Georges Hospital, Blackshaw Road, Tooting, London SW17 0QT, UK

2 Paediatric Physiotherapy Department, St Georges Hospital, London, UK

3 St Georges University of London, London, UK

\section{Introduction}

\section{Problem description}

The physiotherapy run southwest London regional CTEV Ponseti service at St George's Hospital is under the care of the paediatric orthopaedic service. As part of the Ponseti treatment pathway, most patients will require an Achilles tenotomy [1]. Standard practice for the department was for this procedure to be done in theatre under general anaesthetic. The pressures on the health service from COVID-19 reduced theatre capacity, and therefore, the department required a policy change to allow for the continuation of the service. The original setup of tenotomies for CTEV performed in theatre was overdue for a review and the capacity situation allowed for a more effectual change. 


\section{Available knowledge}

CTEV, also known as clubfoot, is the most common congenital birth deformity throughout the world. The majority of cases are idiopathic and consists of midfoot cavus, forefoot adduction and hindfoot equinus and varus [2]. CTEV occurs in 1-2 in 1000 live births and if left untreated can impact a child's development and result in functional impairments [2-4]. The gold-standard for primary correction is the Ponseti method, which consists of serial manipulation and casting. This has been reported to have over a $90 \%$ primary correction rate $[3,5,6]$. In $80-90 \%$ of cases, an Achilles tenotomy is necessary to correct the equinus deformity $[3,7]$.

Practice differs between centres as to the location and anaesthetic protocol for the tenotomy [3, 8-10]. There is evidence to support both local anaesthesia (LA) and general anaesthetic (GA) for the procedure. GA has shown to be safe and provide good analgesic control for the patient [10]. Parada et al. reported the benefits of performing the procedure in theatre under a GA to be better analgesia for the patient and potentially better control of performing the tenotomy. They confirmed the relative safety of a GA in this group of patients but also stated there is uncertainty regarding the long-term effects of GA in infants [10]. MacNeille et al. reported that by performing the procedure under GA techniques such as mini-open can be employed to allow visualisation of the Achilles tendon and avoidance of injury of the neurovascular structures [11]. Other studies have used sedation protocols in theatre to complete tenotomies but avoid using airway management of a GA. Sedation with propofol has been described by multiple centres as safe and effective in clubfoot tenotomy procedures $[12,13]$.

There is good evidence that tenotomies can be performed under LA in the clinic environment. In 2012, Lebel et al. demonstrated low readmission rates after tenotomy under LA [9]. A retrospective analysis comparing Achilles tenotomies under a GA to LA showed there was no statistical difference in terms of recurrence or complications between the two groups [8]. This paper supported the use of LA approach due to reduction in separation time of carers and child, no starvation period and less burden on resources in high turnover centres. Overall, the available literature supports the protocol of performing the Achilles tenotomy under LA in the clinic $[8,9]$.

\section{Rationale}

Prior to the COVID-19 pandemic, patients at St Georges Hospital who required Achilles tenotomy for CTEV were operated on under GA in the operating theatre. The COVID-19 pandemic instigated changes to the previous practice of the paediatric orthopaedic service. National guidance released by The British Orthopaedic Association from March 2020 considered Achilles tenotomies for CTEV to be non-urgent and that the delay in CTEV management should be weighed against the risk of COVID 19 for the patients and families [14]. This resulted in a short cessation of the Ponseti service at St Georges Hospital before the introduction of a new expedited Standard Operating Procedure (SOP) for CTEV patients to have their tenotomies in an outpatient setting. This policy change avoided a delay in the patient's treatment and as a consequence prevented a backlog of patients and kept the risk of COVID-19 infection to the patient and family to a minimum.

\section{Specific aims}

The aim of this project was to assess the safety and feasibility of the percutaneous Achilles tenotomies to be performed under a local anaesthetic in the clinic setting and the parent satisfaction with this service.

\section{Methods}

\section{Context}

This project was prospectively registered as a quality improvement project at St Georges Hospital, London (AUDI000712.). No ethics approval was deemed necessary as the carers were interviewed as a part of the routine clinical visit.

The SOP was developed by a senior physiotherapist, advanced CTEV practitioner and two Consultant paediatric orthopaedic surgeons. The SOP was approved by the COVID-19 infection control, physiotherapy and trauma and orthopaedic care group lead and service managers. It included safety measures in terms of infection control, response to anaphylaxis and uncontrolled bleeding.

The SOP affirmation followed a pilot study on four patients. The tenotomies carried out during the pilot went ahead as planned without complication and with high carer satisfaction. Following that the SOP was deemed feasible and the team continued.

\section{Intervention}

All consecutive patients with a diagnosis of CTEV treated according to the Ponseti method requiring Achilles tenotomy, age less than 6 months with no significant co-morbidity, were included. 
Table 1 Patient demographics and tenotomy characteristics

\begin{tabular}{ll}
\hline Number of patients (feet) & $25(36)$ \\
\hline Male (\%) & $15(60 \%)$ \\
Bilateral (\%) & $13(52 \%)$ \\
Idiopathic (\%) & $19(75 \%)$ \\
Median age at tenotomy (range) & 9 weeks $(4-22)$ \\
Median Pirani score at birth (range) & $4.5(2.5-5)$ \\
Median Pirani score pre-tenotomy (range) & $1.5(0.5-3)$ \\
Median Pirani score post-tenotomy (range) & $0.5(0-1.5)$ \\
\hline
\end{tabular}

For every Achilles tenotomy, a paediatric orthopaedic consultant obtained parental consent and completed an operation note. The tenotomy was carried out under sterile conditions in the Ponseti clinic without the parent present in the room. For each procedure, two physiotherapy practitioners were present to assist the surgeon. The patients received topical cream containing lidocaine and prilocaine prior to the procedure and subcutaneous Chirocaine $0.25 \%$ local anaesthetic. Following the procedure and the casting, the patients were observed for an hour before discharge. Parents were advised regarding the use of post-operative analgesia consisting of regular oral paracetamol. All participant parents received a follow-up phone call by one of the physiotherapists 1 day following their procedure to identify any issues and to assess how the patient was recovering.

All patients were treated as per the pre-COVID-19 local guidelines including consent, indication for tenotomy, casting and post-operative care.

\section{Measures and analysis}

All participants were consecutively recruited from June 11, 2020, until April 12, 2021.
Data was prospectively collected and included: patient demographics, underlying diagnosis, Pirani score at birth and every appointment, evertor muscle activity and any complications during correction.

To assess treatment acceptability and parental experiences, all parents completed a questionnaire following the tenotomy. The SQUIRE guidelines aimed at improving the quality, safety and value of healthcare were used for reporting [15].

Descriptive statistics were used for analysis.

\section{Results}

From June 11, 2020, to April 12, 2021, 36 tenotomies were performed on 25 patients. The results are presented in Table 1. Ages at tenotomy ranged from four to 22 weeks. There were 15 (60\%) male patients. Of the 25 patients, 19 (75\%) were idiopathic and 13 (52\%) were bilateral. None of the patients had an underlying medical condition. No patients were excluded. There were no complications or readmissions as a result of the tenotomies and no patients had to return to clinic for any reason before their arranged appointment. Post-tenotomy, all patients achieved the aimed outcome of ankle dorsiflexion of $15^{\circ}$ or greater. No patients or parents experienced any COVID-19 related complications during the follow-up period. This was confirmed with a follow-up phone call on day one and day seven following the procedure and with the standard hospital infection control measures of temperature measurements and symptomrelated questions completed at the first follow-up in clinic. All the parents completed the parents' perception questionnaire. The results are presented in Table 2. All parents felt their overall experience was positive. All felt that they received enough information before the tenotomy and $96 \%$ felt prepared for the procedure on the day. One parent did
Table 2 A summary of questionnaire responses

\begin{tabular}{lll}
\hline Questions & Yes & No \\
\hline Are you the mother/father/carer? & 25 & 0 \\
Did you receive enough information before the tenotomy? & 25 & 0 \\
Did you feel prepared for the day? & 24 & 1 \\
Would you have been more anxious about having the tenotomy done in an operating & 24 & 1 \\
$\quad$ theatre compared to clinic? & & \\
Did your child experience any pain /complications you were not prepared for? & 25 & 0 \\
Have you received a point of contact in case of need? & 25 & 0 \\
Was your overall experience positive? & 2 & 23 \\
Would you have changed anything? If so what?* & 1 & 24 \\
Would you have preferred to wait a few months until COVID was better controlled? & 0 & 25 \\
Would you have preferred to have the tenotomy done in theatre? & & 25 \\
\hline
\end{tabular}

*Response to what would be different included carers preferred to be in the room whilst procedure took place 
not feel prepared on the day as they were anxious about the possible complications, despite being well informed. None of the parents would have preferred to have the tenotomy completed in theatre and $96 \%$ reported they would have been more anxious about having the procedure done in an operating theatre under GA. One parent stated they had no preference of location. One parent said they would have preferred to wait until COVID-19 was better controlled in the population, whilst 19 parents were happy that the procedure could be done promptly in the clinic. Two parents would have changed parts of the process if they could, both stating they would have liked to be in the room with the child at the time of the procedure.

\section{Discussion}

\section{Summary}

This study demonstrates the safety, efficacy and feasibility of percutaneous Achilles tenotomy done under LA in an outpatient clinic. The results additionally demonstrate excellent parent satisfaction.

\section{Interpretation}

The safety of Achilles tenotomies done under LA has been previously reported $[8,9]$. However, there is a paucity of literature investigating parental treatment acceptability and overall experience of Achilles tenotomies done under LA in a clinic setting. This study found that Achilles tenotomy done under LA is preferable to under GA from a parental perspective. This is supported by previous literature describing parental anxiety surrounding GA in paediatric surgery $[16,17]$. A study looking at anxiety scores of over 200 carers whose children were undergoing surgical procedures displayed high anxiety levels associated with the anaesthetic [17].

With all GAs, there are well-recognised short-term risks but the effects of a GA on the developing brain of the very young patient remains an unsettled topic. Immediate or shortterm serious complications are rare but include respiratory critical events (3.1\%), cardiac instability (1.9\%) and all-cause mortality of 10 in 10,000 as reported in the APRICOT trial, which analysed 31127 anaesthetic procedures for ENT surgery [18]. There is some evidence that GA during infancy has long-term neurodevelopmental implications [19-21]. A systematic review and meta-analysis carried out by Zhang $\mathrm{H}$ et al. concluded that a single GA in children under the age of three moderately increased the risk of developing neurodevelopmental abnormalities. This study included 13 papers for the final analysis and commented that the evidence is poor and further prospective trials are required to make more definitive conclusions. However, there are two trials comparing children's neurodevelopment after being exposed to either a GA or regional anaesthetic for a surgical procedure and no difference was demonstrated [19, 20]. By carrying out Achilles tenotomies for infants under LA, both the known and potential risks of a GA are avoided.

Completing the procedure under LA did not impact the primary outcome of the surgical procedure. Dorsiflexion of the ankle of $15^{\circ}$ or more was achieved in all patients. Our results are consistent with previous studies that report no difference in the requirement for short-term intervention between LA and GA for Achilles tenotomies [8]. Whilst the clinical effectiveness is supported by our study and the literature, there is also a financial benefit for shifting to a clinic procedure. A study from the University of Michigan Hospital in America quantified the cost difference as a saving of $\$ 4121.7$ when performing the tenotomy in the clinic rather than theatre [22].

The COVID-19 pandemic has had unprecedented adverse consequences on healthcare in the UK. Paediatric surgery was specifically impacted by a drastic reduction of operating availability and consequently resulted in a significant backlog [23, 24]. Whilst it was recognised that there would be an impact on the functional outcome of CTEV patients by delays in treatment, national and local protocols were implemented to place a hold on their treatment [24, 25]. Furthermore, when procedures were done in theatre during the pandemic, children and their parents were required to self-isolate for 14 days before the procedure as well as provide a negative COVID-19 swab result 72 hours before the procedure. As only two patients were being scheduled for each theatre session due to infection control measures, these 20 cases sum up to ten potential theatre lists that could be utilised for other cases during the pandemic. In addition, all patients and their parents were spared from 14 days of isolation before the procedure, thus avoiding the adverse physical, psychological and economic consequences that prolonged periods of isolation have been shown to cause $[26,27]$. The feedback from the parents in our study demonstrated that the majority did not wish to delay their child's treatment due to the pandemic and the overall experience resulted in less anxiety when compared with the prospect of the procedure in theatre. The Healthcare Safety Investigation Branch (HSIB) issued a report on the factors behind hospital acquired covid-19 infections in England during the first wave [28]. We have acquired better knowledge from research and guidelines and better Personal Protective Equipment (PPE) but the covid infection officially classified as 'hospital acquired' was significant throughout this time period.

Data regarding the risk of hospital acquired infection in our particular hospital during that period is not available, but the short time spent in the outpatient clinic compared to a day that included pre- and post-op time on the ward, as well as the procedure itself done in theatre, would have 
potentially exposed the patients and parents to more risk of exposure. Our results found no cases of hospital acquired covid infections to any of the patients or families.

\section{Limitations}

The main limitation of this study is the lack of a control group of patients who were treated under GA in theatre. Obtaining a control group for this project was considered both unethical and non-feasible. Retrospectively obtaining results from a previous cohort of patients treated in theatre were not considered suitable due to the time that had passed which would invalidate parents' responses.

\section{Conclusion}

This study found that the new service developed for percutaneous Achilles tenotomies done under LA in an outpatient clinic setting is safe, effective and feasible. This is the first study to describe excellent parent satisfaction with a LA tenotomy. There was no detriment to patients in terms of clinical outcome; there were excellent parents' acceptability and good infection control during the COVID-19 pandemic. Additionally, it freed a significant number of theatre lists and allowed limited resources to be used more efficiently and effectively. This study demonstrates a service improvement opportunity as a result of the COVID-19 pandemic. The success of this project resulted in the continuation of this service beyond the pandemic and is recommended as the standard of care.

Supplementary Information The online version contains supplementary material available at https://doi.org/10.1007/s00264-021-05119-w.

Author contribution Y. Gelfer and T. McNally conceptualised the study, developed the study protocol and participated in its design. A. Russell, A. Bridgens, Y. Gelfer and B. Barkham were involved in recruiting participants to complete the questionnaires. Y. Gelfer and B. Berkham involved in data analysis. Y. Gelfer, T. McNally and B. Barkham contributed to writing and editing the manuscript. A. Bridgens contributed to editing the manuscript. All authors edited the manuscript and read and approved the final version.

Data availability All data was stored in a password-protected Excel spreadsheet.

\section{Declarations}

Ethics approval Consultation with the institutional R\&D offices has deemed this project as a 'patient service evaluation study' with no requirement for ethical approval. Registration and approval for audit and service evaluation were granted (AUDI000712). This study is in compliance with the Helsinki declaration.
Consent to participate No informed consent was deemed necessary.

Consent for publication All the authors have given consent for publication.

Competing interests The authors declare no competing interests.

\section{References}

1. Little Z, Yeo A, Gelfer Y (2019) Poor evertor muscle activity is a predictor of recurrence in idiopathic clubfoot treated by the ponseti method. J Pediatr Orthop 39:e467-71. https://doi.org/10. 1097/BPO.0000000000001357

2. Dobbs MB, Gurnett CA (2009) Update on clubfoot: etiology and treatment. Clin Orthop Relat Res 467:1146-1153. https://doi.org/ 10.1007/s11999-009-0734-9

3. Göksan SB, Bursalı A, Bilgili F, Sıvacıoğlu S, Ayanoğlu S (2006) Ponseti technique for the correction of idiopathic clubfeet presenting up to 1 year of age. A preliminary study in children with untreated or complex deformities. Arch Orthop Trauma Surg 126:15-21. https://doi.org/10.1007/s00402-005-0070-9

4. Hughes K, Gelfer Y, Cokljat M, Wientroub S, Yavor A, Hemo Y et al (2019) Does idiopathic congenital talipes equinovarus have an impact on attainment of developmental milestones? A multicentre international study. J Child Orthop 13:353-360. https://doi. org/10.1302/1863-2548.13.190060

5. Radler C (2013) The Ponseti method for the treatment of congenital club foot: review of the current literature and treatment recommendations. Int Orthop 37:1747-1753. https://doi.org/10. 1007/s00264-013-2031-1

6. Gelfer Y, Wientroub S, Hughes K, Fontalis A, Eastwood DM (2019) Congenital talipes equinovarus: a systematic review of relapse as a primary outcome of the Ponseti method. Bone Joint J 101-B:639-45. https://doi.org/10.1302/0301-620X.101B6.BJJ2018-1421.R1

7. Miller NH, Carry PM, Mark BJ, Engelman GH, Georgopoulos G, Graham S et al (2016) Does strict adherence to the ponseti method improve isolated clubfoot treatment outcomes? A Two-institution Review. Clin Orthop Relat Res 474:237-243. https://doi.org/10. 1007/s11999-015-4559-4

8. Tuhanioğlu Ü, Oğur HU, Seyfettinoğlu F, Çiçek H, Tekbaş VT, Kapukaya A (2018) Percutaneous achillotomy in the treatment of congenital clubfoot: should it be performed in the operating theater or the polyclinic? J Orthop Surg Res 13:155. https://doi. org/10.1186/s13018-018-0851-9

9. Lebel E, Karasik M, Bernstein-Weyel M, Mishukov Y, Peyser A (2012) Achilles tenotomy as an office procedure. J Pediatr Orthop 32:412-415. https://doi.org/10.1097/BPO.0b013e31825611a6

10. Parada CSA, Baird GO, Auffant RA, Tompkins BJ, Caskey PM (2009) Safety of percutaneous tendoachilles tenotomy performed under general anesthesia on infants with idiopathic clubfoot. J Pediatr Orthop 29:916-919. https://doi.org/10.1097/BPO.0b013 e3181c18ab5

11. MacNeille R, Hennrikus W, Stapinski B, Leonard G (2016) A mini-open technique for Achilles tenotomy in infants with clubfoot. J Child Orthop 10:19-23. https://doi.org/10.1007/ s11832-016-0710-3

12. Iravani M, Chalabi J, Kim R, Ebramzadeh E, Zionts LE (2013) Propofol sedation for infants with idiopathic clubfoot undergoing percutaneous tendoachilles tenotomy. J Pediatr Orthop 33:59-62. https://doi.org/10.1097/BPO.0b013e318267037a 
13. Bor N, Katz Y, Vofsi O, Herzenberg JE, Zuckerberg AL (2007) Sedation protocols for Ponseti clubfoot Achilles tenotomy. J Child Orthop 1:333-5. https://doi.org/10.1007/s11832-007-0059-8

14. https://www.boa.ac.uk/uploads/assets/ee39d8a8-9457-4533-9774e 973c835246d/4e3170c2-d85f-4162-a32500f54b1e3b1f/COVID19-BOASTs-Combined-FINAL.pdf. Accessed 10/09/2020. British Orthopaedic Association. Management of patients with urgent orthopaedic conditions and trauma during the coronavirus pandemic. BOA 2020;1

15. Ogrinc G, Davies L, Goodman D, Batalden P, Davidoff F, Stevens D (2016) SQUIRE 2.0 ( Standards for QUality Improvement Reporting Excellence): revised publication guidelines from a detailed consensus process: Table 1. BMJ Qual Saf 25:986-992. https://doi.org/10.1136/bmjqs-2015-004411

16. Shirley PJ, Thompson N, Kenward M, Johnston G (1998) Parental anxiety before elective surgery in children A British perspective. Anaesthesia 53:956-9. https://doi.org/10.1046/j.1365-2044.1998. 00533.x

17. Ayenew NT, Endalew NS, Agegnehu AF, Bizuneh YB (2020) Prevalence and factors associated with preoperative parental anxiety among parents of children undergoing anesthesia and surgery: A cross-sectional study. Int J Surg Open 24:18-26. https://doi.org/ 10.1016/j.ijso.2020.03.004

18. Habre W, Disma N, Virag K, Becke K, Hansen TG, Jöhr M et al (2017) Incidence of severe critical events in paediatric anaesthesia (APRICOT): a prospective multicentre observational study in 261 hospitals in Europe. Lancet Respir Med 5:412-425. https://doi. org/10.1016/S2213-2600(17)30116-9

19. Davidson AJ, Disma N, de Graaff JC, Withington DE, Dorris L, Bell G et al (2016) Neurodevelopmental outcome at 2 years of age after general anaesthesia and awake-regional anaesthesia in infancy (GAS): an international multicentre, randomised controlled trial. Lancet 387:239-250. https://doi.org/10.1016/S01406736(15)00608-X

20. McCann ME, de Graaff JC, Dorris L, Disma N, Withington D, Bell G et al (2019) Neurodevelopmental outcome at 5 years of age after general anaesthesia or awake-regional anaesthesia in infancy (GAS): an international, multicentre, randomised, controlled equivalence trial. Lancet 393:664-677. https://doi.org/10. 1016/S0140-6736(18)32485-1
21. Zhang H, Du L, Du Z, Jiang H, Han D, Li Q (2015) Association between childhood exposure to single general anesthesia and neurodevelopment: a systematic review and meta-analysis of cohort study. J Anesth 29:749-757. https://doi.org/10.1007/ s00540-015-2030-z

22. Hedrick B, Gettys FK, Richards S, Muchow RD, Jo CH, Abbott MD (2018) Percutaneous heel cord release for clubfoot: a retrospective, multicentre cost analysis. J Child Orthop 12:273-278. https://doi.org/10.1302/1863-2548.12.170216

23. Macdonald N, Clements C, Sobti A, Rossiter D, Unnithan A, Bosanquet N (2020) Tackling the elective case backlog generated by Covid-19: the scale of the problem and solutions. J Public Health 42:712-716. https://doi.org/10.1093/pubmed/fdaa155

24. Green, G, Eastwood, D.M., Hashemi-Nejad, A, Calder, P, Tennent, S, Khan, T, et al (2020) COVID-19 and resuming elective paediatric orthopaedics in a London major trauma centre and a tertiary referral centre. BOA Online

25. UK Clubfoot Consensus Group (2020) Management of clubfoot infants and children during the Covid-19 pandemic. https:// www.c-ProdirectCom/Content/Files/UKCCG\%20Clubfoot\% 20and\%20Covid-19Pdfn.d

26. Nicola M, Alsafi Z, Sohrabi C, Kerwan A, Al-Jabir A, Iosifidis C et al (2020) The socio-economic implications of the coronavirus pandemic (COVID-19): A review. Int J Surg 78:185-193. https:// doi.org/10.1016/j.ijsu.2020.04.018

27. White RG, Van der Boor C (2020) Impact of the COVID-19 pandemic and initial period of lockdown on the mental health and well-being of adults in the UK. BJPsych Open 6(e):90. https:// doi.org/10.1192/bjo.2020.79

28. Healthcare Safety Investigation Branch. National report charts challenges of managing COVID-19 transmission in hospitals. 29 Oct 2020. https://www.hsib.org.uk/news/national-report-chartschallenges-managing-covid-19-transmission-hospitals/

Publisher's note Springer Nature remains neutral with regard to jurisdictional claims in published maps and institutional affiliations. 